\title{
DEFICIÊNCIA DE MACRONUTRIENTES E DE BORO EM ABACAXIZEIRO 'IMPERIAL': COMPOSIÇÃO MINERAL' ${ }^{1}$
}

\author{
MARIA JOSÉ MOTA RAMOS 2 , PEDRO HENRIQUE MONNERAT ${ }^{3}$, \\ LEANDRO GLAYDSON DA ROCHA PINHO ${ }^{4}$, JOSÉ ACCÁCIO DA SILVA 5
}

RESUMO- Avaliaram-se, neste trabalho, os efeitos das deficiências dos macronutrientes e do boro em abacaxizeiro 'Imperial' sobre os teores foliares desses nutrientes. Os tratamentos Completo, - N; - P;- K; $\mathrm{Ca}$; - Mg; - S e - B foram aplicados como soluções nutritivas, em vasos plásticos com $14 \mathrm{~kg}$ de areia de praia purificada e uma muda de abacaxi como unidade experimental. O delineamento foi em blocos casualizados completos, com seis repetições. Determinaram-se os teores de N, P, K, Ca, Mg, S e B na folha "D" inteira aos cinco, sete, nove e 12 meses após o plantio. A deficiência de $\mathrm{N}$ reduziu a absorção de $\mathrm{S}$, mas aumentou os teores de $\mathrm{P}, \mathrm{K}, \mathrm{Mg}$ e $\mathrm{B}$; a de $\mathrm{P}$ reduziu os teores de $\mathrm{N}, \mathrm{Ca}, \mathrm{Mg}, \mathrm{S}$ e B, mas aumentou os de $\mathrm{K}$; a de $\mathrm{K}$ aumentou os teores de N, P, Ca, Mg, S e B; a de Ca aumentou os teores de $\mathrm{K}$ e $\mathrm{Mg}$ e reduziu os de $\mathrm{S}$; as de $\mathrm{Mg}$ e de $\mathrm{B}$ aumentaram os teores de $\mathrm{Ca}$, e a de $\mathrm{S}$ aumentou os teores de $\mathrm{K}$ e $\mathrm{B}$. Os teores foliares das plantas normais e deficientes, no início da formação dos frutos, foram, respectivamente: $\mathrm{N}=14,8$ e 9,7; $\mathrm{P}=1,23$ e 0,67; $\mathrm{K}=20,0$ e 11,6; Ca: 4,37 e 0,91; $\mathrm{Mg}=2,26$ e 0,$73 ; \mathrm{S}=1,45$ e $0,45 \mathrm{~g} \mathrm{~kg}^{-1}$ e $\mathrm{B}=18,4$ e $5,8 \mathrm{mg} \mathrm{kg}^{-1}$.

Termos para indexação: diagnose foliar, teor de nutrientes, solução nutritiva.

\section{MACRONUTRIENTS AND BORON DEFICIENCY IN 'IMPERIAL' PINEAPPLE: MINERAL COMPOSITION}

\begin{abstract}
This study was carried out to evaluate the effects of macronutrients and boron deficiencies in 'Imperial' pineapple on the leaf concentrations of those nutrients. The treatments Complete, - N, - P; - K, - $\mathrm{Ca},-\mathrm{Mg},-\mathrm{S}$ and - B were applied as nutrient solutions, to plastic pots filled with $14 \mathrm{~kg}$ of purified beach sand and one pineapple seedling, as the experimental unit, in a randomized complete block design with six replicates. The " $\mathrm{D}$ " leaf concentrations of $\mathrm{N}, \mathrm{P}, \mathrm{K}, \mathrm{Ca}, \mathrm{Mg}, \mathrm{S}$ and B were determined at five, seven, nine and twelve months after the planting. The deficiency of $\mathrm{N}$ reduced the absorption of $\mathrm{S}$, but it increased the concentrations of $\mathrm{P}, \mathrm{K}, \mathrm{Mg}$ and $\mathrm{B}$; that of $\mathrm{P}$ reduced $\mathrm{N}, \mathrm{Ca}, \mathrm{Mg}, \mathrm{S}$ and $\mathrm{B}$ concentrations, but it increased that of $\mathrm{K}$; that of $\mathrm{K}$ increased $\mathrm{N}, \mathrm{P}, \mathrm{Ca}, \mathrm{Mg}, \mathrm{S}$ and $\mathrm{B}$ concentrations; that of $\mathrm{Ca}$ increased $\mathrm{K}$ and $\mathrm{Mg}$ concentrations and reduced the one of $\mathrm{S}$; that of $\mathrm{Mg}$ and of $\mathrm{B}$ increased $\mathrm{Ca}$ concentration and that of $\mathrm{S}$ increased $\mathrm{K}$ and $\mathrm{B}$ concentrations. The leaf concentrations of the normal and deficient plants at the beginning of fruit growth were, respectively: $\mathrm{N}=14.8$ and $9.7 ; \mathrm{P}=1.23$ and $0.67 ; \mathrm{K}=20.0$ and $11.6 ; \mathrm{Ca}: 4.37$ and $0.91 ; \mathrm{Mg}=2.26$ and $0.73 ; \mathrm{S}=1.45$ and $0.45 \mathrm{~g} \mathrm{~kg}^{-1}$ and $\mathrm{B}=18.4$ and $5.8 \mathrm{mg} \mathrm{kg}^{-1}$.
\end{abstract}

Index terms: leaf diagnosis; nutrient content; nutrient solution.

\footnotetext{
${ }^{1}$ (Trabalho 036-10). Recebido em: 25-10-2010. Aceito para publicaçaõ em: 22-04-2010. Parte da tese de doutorado em Produção Vegetal,UENF/RJ, do primeiro autor.

${ }^{2}$ Pesquisadora da EMPAER/MT/COPESQ, Rua Jari Gomes, n. 454, Boa Esperança, Cuiabá-MT, E-mail: majumota@ig.com.br

${ }^{3}$ Professor titular UENF/CCTA/LFIT. E-mail: monnerat@uenf.br

${ }^{4}$ Professor do IFES. E-mail: leandro.pinho@ifes.edu.br

${ }^{5}$ Engenheiro químico UENF/CCTA/LFIT. E-mail: jaccacio@uenf.br
} 


\section{INTRODUÇÃO}

$\mathrm{O}$ estado nutricional do abacaxizeiro tem uma grande influência no crescimento da planta e, consequentemente, na produção e na qualidade do fruto (MALÉZIEUX; BARTHOLOMEW, 2003).

No abacaxizeiro, as folhas ' $\mathrm{D}$ ', as mais jovens entre as adultas, formam um ângulo de $45^{\circ}$ com o solo e são facilmente identificáveis, sendo comumente usadas para avaliar o índice de crescimento e o estado nutricional da planta. Comparando os valores da concentração foliar dos nutrientes de uma amostra-problema com os valores-padrão publicados em tabelas, ou verificados em plantas normais, constata-se se há ou não deficiência dos nutrientes analisados (FONTES, 2001). A diagnose foliar baseia-se na premissa de existir uma relação direta entre o suprimento de nutrientes e os teores desses nutrientes na planta, e que aumentos ou decréscimos nas concentrações se relacionam com produção mais alta ou mais baixa (BATAGLIA; SANTOS, 2001).

A quantidade total de nutrientes na planta aumenta com a idade, mas a concentração em um dado tecido pode aumentar, diminuir ou permanecer inalterada, dependendo do nutriente e do tecido. Mudanças sazonais nas concentrações do nutriente no tecido ocorrem, mas, segundo Malézieux e Bartholomew (2003), elas podem resultar de mudanças no crescimento da planta a uma taxa maior do que as de mudanças na disponibilidade do nutriente para a planta.

Para o cv. Smooth Cayenne, existem informações sobre os teores adequados de nutrientes, evitando que deficiências causem baixa produtividade e pior qualidade dos frutos e excessos, desperdícios e perigos à qualidade ambiental. Tais informações são necessárias para outras cultivares e para alguns novos híbridos que têm exigências nutricionais diferentes daquelas do'Smooth Cayenne'.

O abacaxizeiro tem pequena demanda de nitrogênio e potássio até, aproximadamente, quatro meses após o plantio; depois, a necessidade aumenta com o crescimento até a indução floral (MALÉZIEUX; BARTHOLOMEW, 2003). Esses são os nutrientes exigidos em maiores quantidades (PAULA et al., 1998; MALÉZIEUX; BARTHOLOMEW, 2003). A necessidade de cálcio é pequena, enquanto a de magnésio é intermediária (PY; TISSEAU citados por PAULA et al., 1999). Potássio acumula-se nas folhas em uma quantidade maior que qualquer outro nutriente. Nitrogênio promove crescimento vegetativo vigoroso e assim exerce grande influência no peso do fruto, enquanto o potássio interfere principalmente na qualidade
(SOUZA; REINHARDT, 2004).

O objetivo deste trabalho foi avaliar os efeitos das deficiências dos macronutrientes e do boro nos teores foliares desses nutrientes em quatro fases do ciclo da planta do abacaxizeiro 'Imperial'.

\section{MATERIAL E MÉTODOS}

O experimento foi implantado em casa de vegetação do Setor de Nutrição Mineral de Plantas da UENF, em Campos dos Goytacazes-RJ, no dia 19 de dezembro de 2003, utilizando-se de mudas do abacaxizeiro 'Imperial' produzidas a partir de cultura de tecidos na Campo Biotecnologia Vegetal Ltda., Unidade Biofábrica/EMBRAPA-CNPMF. O experimento constou de oito tratamentos: Completo, -N, -P, -K, -Ca, -Mg, -S e -B, em blocos casualizados completos, com seis repetições. A unidade experimental constou de um vaso plástico contendo $14 \mathrm{~kg}$ de areia de praia previamente purificada, contendo uma muda de abacaxizeiro. A purificação consistiu na embebição da areia com ácido clorídrico diluído em água, na proporção de 1:4 durante cerca de quatro horas, em recipiente plástico de 60 litros e posterior lavagem com água pura até que o $\mathrm{pH}$ se estabilizasse em valor próximo de 5, quando, então, fez-se uma lavagem final com água desionizada. As mudas tinham tamanho médio de $6 \mathrm{~cm}$ e, após o plantio, receberam $500 \mathrm{~mL}$ de água desionizada a cada dois dias, durante 15 dias, quando novas raízes começaram a ser emitidas. Todos os vasos passaram, então, a receber, três vezes por semana, $500 \mathrm{~mL}$ da solução completa, que apresentava a seguinte composição, em mg L-1: $\mathrm{N}\left(\mathrm{NO}_{3}^{-}\right)=112 ; \mathrm{N}\left(\mathrm{NH}_{4}^{+}\right)=3,5 ; \mathrm{P}=7,74$; $\mathrm{K}=156,4 ; \mathrm{Ca}=80 ; \mathrm{Mg}=24,3 ; \mathrm{S}=32 ; 0 ; \mathrm{Cl}=1,77$; $\mathrm{Mn}=0,55 ; \mathrm{Zn}=0,13 ; \mathrm{Cu}=0,03 ; \mathrm{Mo}=0,05 ; \mathrm{B}=0,27$; $\mathrm{Fe}=2,23 \mathrm{com} \mathrm{pH}=5,5$. Após 50 dias de aplicação da solução completa ou 65 dias após o transplante, induziu-se a deficiência de boro, subtraindo-o dos vasos que receberam esse tratamento. Os demais tratamentos continuaram a receber a solução completa até 105 dias após o transplante, quando, então, reduziu-se a concentração do macronutriente a 10\% da solução completa. Aos 150 dias após o transplante, os tratamentos $-\mathrm{N},-\mathrm{P},-\mathrm{K},-\mathrm{Ca},-\mathrm{Mg}$ e $-\mathrm{S}$ tiveram seus respectivos nutrientes totalmente subtraídos da solução (Tabela 1). Aos 240 dias após o transplante, o tratamento $-\mathrm{N}$ voltou a receber solução nutritiva com $10 \%$ de $\mathrm{N}$ da solução completa, por 12 vezes, para evitar que as plantas ficassem impedidas de frutificar, futuramente. Os volumes de solução nutritiva aplicados variaram com o porte das plantas, sendo $500 \mathrm{~mL}$ até 150 dias após o plantio; $700 \mathrm{~mL}$, de 150 até 330 dias, e $1 \mathrm{~L}$ até a colheita, aplicados 
três vezes por semana.

Todas as plantas foram induzidas ao florescimento oito meses após o plantio, quando as folhas " $D$ ", no tratamento completo, atingiram, em média, $63,4 \mathrm{~cm}$ de comprimento e com um número total de folhas de 55. Aplicaram-se, na roseta foliar, por planta, $50 \mathrm{~mL}$ da solução de Etrel $0,1 \%$, ureia $2 \%$ e de hidróxido de cálcio 0,035\% (VELOSO et al., 2001).

Amostragens foliares foram feitas aos cinco, sete, nove e 12 meses após o plantio, coletando-se uma folha " $D$ " de cada planta, para avaliação dos teores de $\mathrm{N}, \mathrm{P}, \mathrm{K}, \mathrm{Ca}, \mathrm{Mg}, \mathrm{S}$ e B.

$\mathrm{Na}$ determinação dos teores de nutrientes, foi utilizada a folha "D" inteira (SIEBENEICHLER, 2002). A coleta dessa folha foi realizada na parte da manhã, entre nove e 10 horas. As folhas foram limpas com algodão umedecido com água desionizada e secas em estufa de circulação forçada de ar a $70-75^{\circ} \mathrm{C}$, por 72 horas. Depois de secas, elas foram pesadas e moídas em moinho tipo Wiley, passadas em peneira de 20 mesh e armazenadas em frascos hermeticamente fechados. $\mathrm{O} N$ foi determinado pelo método de Nessler (JACKSON, 1965), após digestão sulfúrica $\left(\mathrm{H}_{2} \mathrm{SO} 4\right.$ e $\left.\mathrm{H}_{2} \mathrm{O}_{2}\right)$ do tecido vegetal. P foi determinado, colorimetricamente, pelo método do azul de molibdênio; $\mathrm{K}$, por fotometria de chama; $\mathrm{Ca}$ e Mg, por espectrofotometria de absorção atômica, e S, por turbidimetria de sulfato, no extrato da digestão nitro-perclórica $\left(\mathrm{HNO}_{3}\right.$ e $\left.\mathrm{HClO}_{4}\right)$, e B foi determinado colorimetricamente pela azometina $\mathrm{H}$, após incineração em mufla (EMBRAPA, 1999).

Os dados foram submetidos à análise de variância como um arranjo fatorial $8 \times 4$ (oito tratamentos $\mathrm{x}$ quatro épocas de coleta). As médias dos tratamentos sob deficiência foram comparadas à do controle, pelo teste de Dunnet, a 5\%, e as médias das épocas, pelo teste de Tukey,a 5\%.

\section{RESULTADOS E DISCUSSÃO}

As deficiências minerais induzidas reduziram o crescimento e promoveram o aparecimento de sintomas característicos nas folhas e nos frutos das plantas de abacaxi (RAMOS et al., 2009). Cada nutriente deficiente reduziu seu próprio teor foliar em todas as quatro épocas analisadas (Tabelas 2 a 8).

\section{Deficiência de nitrogênio}

Os teores de $\mathrm{N}$ do tratamento completo não variaram significativamente durante o ciclo do abacaxizeiro, mas, no tratamento $-\mathrm{N}$, o teor decresceu do quinto para o sétimo mês e aumentou no nono, voltando a cair aos 12 meses (Tabela 2). O aumento verificado aos 9 meses foi devido às 12 aplicações de solução contendo $\mathrm{N}$ efetuadas a partir do oitavo mês, para evitar que as plantas entrassem em colapso e não chegassem a frutificar. Os decréscimos observados no tratamento $-\mathrm{N}$ durante o ciclo são atribuídos à diluição do $\mathrm{N}$ em virtude do crescimento vegetativo e da formação dos frutos.

Os teores de $\mathrm{P}, \mathrm{K}$ e B aumentaram nas plantas submetidas à deficiência de $\mathrm{N}$, e esses aumentos podem ser atribuídos à restrição de crescimento provocada pela deficiência, causando acumulação na planta e, consequentemente, aumento do teor. $\mathrm{O}$ teor de $\mathrm{Mg}$ foi também aumentado pela deficiência de N, mas efeito significativo só foi observado aos 12 meses.

O nitrogênio é componente integral de muitos compostos essenciais aos processos de crescimento vegetal, como os aminoácidos e as proteínas. É indispensável para a utilização de carboidratos no interior da planta; estimula o crescimento e o desenvolvimento de folhas, caules e raízes, e promove maior absorção de outros nutrientes.

Houve diminuição da concentração de $\mathrm{K}$, no tratamento $-\mathrm{N}$, aos nove meses após o plantio em relação ao sétimo mês (Tabela 2). Essa redução coincide com o aumento do teor de $\mathrm{N}$ provocado pelo retorno da aplicação de nitrogênio aos oito meses após o plantio, para evitar o colapso das plantas. $\mathrm{O}$ crescimento promovido por tal aplicação causou a diluição do K na planta. Entretanto, Viegas et al. (2004) observaram que K foliar foi reduzido em plantas de camu-camu (Myrciaria dubia) quando $\mathrm{N}$ estava deficiente.

Não houve influência da deficiência de $\mathrm{N}$ na concentração foliar de $\mathrm{Ca}$, em nenhuma das quatro épocas avaliadas (Tabela 2).

A deficiência de $\mathrm{N}$ reduziu os teores de $\mathrm{S}$ nas folhas, indicando que a absorção desse nutriente foi reduzida pela deficiência de $\mathrm{N}$, diferentemente do que ocorreu com $\mathrm{P}, \mathrm{K}, \mathrm{B}$ e $\mathrm{Mg}$. Essa redução não surpreende, tendo em vista a estreita coordenação entre as assimilações de $\mathrm{Ne} \mathrm{S}$, em que a deficiência de um reprime a assimilação do outro (KOPRIVOVA et al., 2000; NEUENSCHWANDER et al., 1991). É também provável que a síntese do polipepitídeo glutationa, que atua como um sinal que coordena a absorção radicular do sulfato e sua assimilação na parte aérea, tenha sido limitada pela deficiência de N (EPSTEIN; BLOOM, 2006).

Outra possível explicação seria a absorção do $\mathrm{S}$ ter sido deprimida pelo cloreto presente na solução $-\mathrm{N}$, pois, nessa solução, todo o nitrato foi substituído por cloreto.

As concentrações de $\mathrm{N}$ obtidas neste trabalho, no tratamento completo, de 13,3 a $14,8 \mathrm{~g} \mathrm{~kg}^{-1}$, na 
folha inteira, estão um pouco abaixo dos valores citados por Jones et al. (1991) e Boaretto et al. (1999) que variam de $15-17 \mathrm{~g} \mathrm{~kg}^{-1}$ de nitrogênio na porção clorofilada das folhas. Siebeneichler (2002), trabalhando com a cultivar Pérola, verificou uma concentração de $\mathrm{N}$ foliar de $16,3 \mathrm{~g} \mathrm{~kg}^{-1}$, na folha inteira, também um pouco acima do nível da 'Imperial', nesse trabalho. Malavolta et al. (1997) citam um teor de 20-22 $\mathrm{g} \mathrm{kg}^{-1}$ como adequado para o abacaxizeiro na folha inteira ou porção clorofilada, bem acima do encontrado nesse trabalho. É conveniente considerar que, em geral, as faixas adequadas apresentadas pelos diferentes autores implicam que, se as concentrações encontradas estiverem dentro da faixa, a planta não estará deficiente, mas, se estiverem abaixo, não significa que as plantas estejam deficientes. Para serem consideradas deficientes, as plantas devem ter teor menor que o nível crítico ou faixa crítica, informação esta raramente disponível.

Ressalta-se a necessidade de obtenção de níveis ou faixas críticas ou de suficiência em épocas predeterminadas, que melhor representem as exigências para produção do abacaxizeiro. $\mathrm{Na}$ literatura, encontram-se sugestões de diferentes épocas para amostragem (MALAVOLTA et al., 1997; REUTER; ROBINSON, 1988; JONES et al. 1991, BOARETTO et al., 1999). Possivelmente, o período antes da floração (BOARETTO et al. (1999), represente melhor o estado nutricional do abacaxizeiro. Neste trabalho, as plantas foram amostradas em diferentes épocas devido à necessidade de rigoroso acompanhamento do estado nutricional, fato que dificulta a comparação com teores de nutrientes encontrados em épocas não correlatas de outros trabalhos. Diferenças entre cultivares devem, também, ser consideradas.

\section{Deficiência de Fósforo}

No tratamento completo, houve diminuição da concentração do P no décimo segundo mês após o plantio (fase de formação do fruto), em relação ao quinto e ao sétimo mês (Tabela 3 ). A diminuição do teor de $\mathrm{P}$ a partir do sétimo mês após o plantio, provavelmente tenha ocorrido por maior demanda de $\mathrm{P}$ nos processos de florescimento, frutificação e formação de rebentos, causando a diluição desse nutriente na folha pela sua translocação para atender a tais processos.

A deficiência de $\mathrm{P}$ diminuiu a concentração do N, S e B (Tabela 3 ) e, tendo em vista que a deficiência de fósforo reduziu o crescimento das plantas, a absorção desses nutrientes também deve ter sido reduzida. Situação semelhante aconteceu com as concentrações de $\mathrm{Mg}$ e de $\mathrm{Ca}$, mas somente aos 12 meses após o plantio (Tabela 3). A causa da redução da absorção desses nutrientes pode ser atribuída ao papel exercido pelo $P$ nos processos de acumulação e transferência de energia sob a forma de ATP, principalmente. A absorção de nutrientes é um processo que, em geral, demanda energia (MARSCHNER, 1995; EPSTEIN; BLOOM, 2006).

A concentração de $\mathrm{K}$ aos 12 meses após o plantio foi aumentada pela deficiência de P (Tabela 3), e esse aumento pode ser atribuído à restrição do crescimento promovido pela deficiência, levando ao efeito de concentração do potássio.

As concentrações do P, no tratamento completo (Tabela 3), estão um pouco acima daquelas obtidas por Quaggio et al. (1996), que variaram de 0,9-1,1 g $\mathrm{kg}^{-1}$, mas abaixo do valor de $2,09 \mathrm{~g} \mathrm{~kg}^{-1}$ encontrado por Siebeneichler (2002) na folha inteira e de 2,1 a 2,3 g kg-1, na folha D inteira ou porção clorofilada, segundo Malavolta et al. (1997).

\section{Deficiência de Potássio}

Os teores de $\mathrm{K}$ do tratamento completo não variaram significativamente durante o ciclo do abacaxizeiro, mas, no tratamento $-\mathrm{K}$, o teor decresceu do quinto para o sétimo e nono meses e entre esses não houve diferença significativa (Tabela 4). Houve uma diminuição de $87 \%$ no teor de $\mathrm{K}$, sob deficiência, no décimo segundo mês após o plantio, em relação ao completo. Os decréscimos observados no sétimo mês, em relação ao quinto mês, podem ser atribuídos à demanda do $\mathrm{K}$ no período vegetativo e um ano após o plantio, pela maior extração desse nutriente na fase de frutificação.

A deficiência do $\mathrm{K}$ aumentou os teores de $\mathrm{N}$, $\mathrm{Ca}$ e $\mathrm{Mg}$ durante o ciclo da cultura de forma mais consistente (Tabela 4). Esses aumentos podem, em parte, ser atribuídos à redução do crescimento, mas, nos casos de $\mathrm{Ca}$ e $\mathrm{Mg}$, também ao antagonismo existente entre esses nutrientes e o K; na ausência de potássio, $\mathrm{Ca}$ e $\mathrm{Mg}$ têm a absorção aumentada (MALAVOLTA et al., 1997).

Os aumentos nos teores de $\mathrm{P}$ nos períodos de floração e frutificação, e de S e B, no período de frutificação, podem ser atribuídos à redução de crescimento provocada pela deficiência de potássio.

Em gravioleiras (Anona muricata L.), Frazão et al. (2002) observaram que a omissão de K aumentou os teores foliares de Mg e N, e Avilán (1975) e Silva et al. (1986), o teor de N.

Os teores do $\mathrm{K}$ de 21,6 a 23,8 $\mathrm{g} \mathrm{kg}^{-1}$, obtidos no tratamento completo, no presente estudo (Tabela 4), foram próximos daqueles mencionados por Boaretto et al. (1999) e Jones et al. (1991), que variaram de 22 a $30 \mathrm{~g} \mathrm{~kg}^{-1}$, e do valor de 20,4, citado por Siebeneichler (2002), mas acima do teor de $17,3 \mathrm{~g} \mathrm{~kg}^{-1}$ citado por Hiroce et al. (1977). Malavolta et al. (1997) citam a faixa de 25 a $27 \mathrm{~g} \mathrm{~kg}^{-1}$ na matéria seca da folha "D" 
inteira ou em sua porção clorofilada.

\section{Deficiência de Cálcio}

Houve aumento de $74 \%$ no teor do Ca na folha ' $\mathrm{D}$ ' das plantas cultivadas no tratamento completo, aos 12 meses, em relação aos 9 meses após o plantio, período em que ocorreu a frutificação (Tabela 5). Esse aumento do teor foliar pode ser atribuído à paralisação da expansão foliar causada pelo crescimento do fruto, que é um dreno forte para os carboidratos produzidos pela fotossíntese e um dreno fraco para o $\mathrm{Ca}$, cuja mobilidade no floema é baixa (KLUGE et al., 2001). $\mathrm{O}$ Ca movimenta-se preferencialmente pelo xilema e acumula-se principalmente nas folhas por causa de sua maior taxa transpiratória. $\mathrm{O}$ teor foliar de $\mathrm{Ca}$ nas plantas deficientes ficou próximo de $20 \%$ do completo, aos 9 e 12 meses do plantio.

A deficiência do $\mathrm{Ca}$ aumentou o teor do $\mathrm{K}$ aos 7 e 12 meses após o plantio; do $\mathrm{Mg}$, aos 7; 9 e 12 meses, e diminuiu o do S aos 12 meses (Tabela 5). Não houve influência da deficiência do $\mathrm{Ca}$ nos teores foliares do N, P e B em nenhuma das quatro épocas avaliadas.

Os efeitos interiônicos entre $\mathrm{K}, \mathrm{Ca}$ e $\mathrm{Mg}$ são evidentes neste estudo. A ausência de cálcio no substrato anulou a competição que ele exerce sobre a absorção de $\mathrm{K}$ e Mg, aumentando a absorção destes (MALAVOLTA et al., 1997).

As concentrações de $\mathrm{Ca}$ obtidas neste trabalho, no tratamento completo, de 4,30 a 4,40 $\mathrm{g} \mathrm{kg}^{-1}$, na folha inteira, nas épocas que antecederam a frutificação (Tabela 5), estão próximas dos valores citados por Siebeneichler (2002), de 3,92 $\mathrm{g} \mathrm{kg}^{-1}$, e Malavolta et al. (1997), de 3 a $4 \mathrm{~g} \mathrm{~kg}^{-1}$; porém, aos 12 meses, foram bem superiores $\left(7,59 \mathrm{~g} \mathrm{~kg}^{-1}\right)$.

\section{Deficiência de Magnésio}

$\mathrm{O}$ teor de $\mathrm{Mg}$ do tratamento completo foi maior no décimo segundo mês após o plantio, época de menor exigência desse nutriente pelo abacaxizeiro (Tabela 6). A deficiência do $\mathrm{Mg}$ não influenciou nos teores do N, S e B em nenhuma das épocas avaliadas. Os teores de $\mathrm{P}$ e $\mathrm{K}$ aumentaram em apenas uma época de amostragem, mas sem tendência definida. Os teores de $\mathrm{Ca}$, entretanto, tiveram, em geral, substancial aumento na deficiência de $\mathrm{Mg}$, e tal fato pode ser atribuído ao antagonismo entre esses dois nutrientes (MALAVOLTA et al., 1997).

Os teores de Mg obtidos neste trabalho, no tratamento completo, de 2,10 a $3,07 \mathrm{~g} \mathrm{~kg}^{-1}$, na folha inteira, estão próximos dos valores citados por Siebeneichler (2002), de 2,42 $\mathrm{g} \mathrm{kg}^{-1}$, mas abaixo dos valores mencionados por Malavolta et al. (1997), de 4 a $5 \mathrm{~g} \mathrm{~kg}^{-1}$, e por Boaretto et al. (1999), de 3 a $4 \mathrm{~g} \mathrm{~kg}^{-1}$. variaram significativamente durante o ciclo do abacaxizeiro, mas, no tratamento -S houve redução de aproximadamente 53\% no seu teor, aos 7 meses após o plantio, em relação aos 5 meses após o que o teor se manteve razoavelmente estável (Tabela 7).

A deficiência de $\mathrm{S}$ aumentou a concentração de $\mathrm{K}$, aos 7 e 12 meses após o plantio, de $\mathrm{P}$ aos 9 meses e do Ca e B aos 12 meses (Tabela 7). Viegas et al. (2004) encontraram teor foliar de Ca reduzido no camucamuzeiro (Myrciaria dubia) quando o S estava deficiente. Não houve influência da deficiência do $\mathrm{S}$ nos teores do $\mathrm{N}$ e do $\mathrm{Mg}$, em comparação ao completo, mas a concentração do $\mathrm{Mg}$ elevou-se aos 12 meses após o plantio, em relação às outras épocas.

Os teores foliares médios de $\mathrm{S}$ do tratamento completo de 1,6 $\mathrm{g} \mathrm{kg}^{-1}$ são próximos do valor de $1,4 \mathrm{~g} \mathrm{~kg}^{-1}$ obtido por Siebeneichler (2002), em abacaxizeiro 'Pérola', mas bem abaixo da faixa de 2 a $3 \mathrm{~g} \mathrm{~kg}^{-1}$ relatada por Malavolta et al. (1997).

\section{Deficiência de Boro}

$\mathrm{O}$ teor de $\mathrm{B}$ aumentou em $66,0 \%$ no décimo segundo mês após o plantio em relação ao nono mês, no tratamento completo (Tabela 8), de modo semelhante ao que ocorreu com os teores foliares de $\mathrm{Ca}$ (Tabela 5), pois ambos os nutrientes apresentam baixa mobilidade no floema. $\mathrm{O}$ valor mais baixo, de $5,5 \mathrm{~g} \mathrm{~kg}^{-1}$ de $\mathrm{B}$, foi obtido próximo à colheita dos frutos de plantas deficientes de boro (Tabela 8). Siebeneichler (2002) obteve o valor de 4,2 $\mathrm{g} \mathrm{kg}^{-1} \mathrm{na}$ folha "D", em plantas da cultivar 'Pérola', aos dez meses de idade.

A deficiência do $\mathrm{B}$ não alterou os teores de $\mathrm{N}, \mathrm{P}, \mathrm{K}$ e $\mathrm{Mg}$ em nenhuma das quatro épocas analisadas (Tabela 8). Os teores de Ca aumentaram aos 12 meses do plantio por efeito da deficiência de boro, em consequência da redução do crescimento vegetativo da planta e do fato de que os frutos não são um dreno eficiente para cálcio (MARSCHNER, 1995). Embora os teores de $S$ tenham aumentado aos sete meses, em virtude da deficiência de boro, não houve consistência nas demais épocas.

Os teores de B do tratamento completo variaram de 21,2 a 30,5 $\mathrm{g} \mathrm{kg}^{-1}$ e são semelhantes aos valores de 19,3 a 24,1 $\mathrm{g} \mathrm{kg}^{-1}$ obtidos por Siebeneichler (2002) em abacaxizeiro 'Pérola'. Malavolta et al. (1997) consideram a faixa de 30 a $40 \mathrm{~g} \mathrm{~kg}^{-1}$ como adequada para o abacaxizeiro, enquanto Boaretto et al. (1999) mencionam a faixa de 20 a $40 \mathrm{mg} \mathrm{kg}^{-1} \mathrm{de} \mathrm{B}$. 
TABELA 1- Composição química da solução nutritiva completa e com omissão dos macronutrientes e do boro $\left(\mathrm{mL} \mathrm{L}^{-1}\right)$.

\begin{tabular}{ccccccccc}
\hline Soluções estoques & Completa & $-\mathbf{N}$ & $\mathbf{- P}$ & $\mathbf{- K}$ & $\mathbf{- C a}$ & $\mathbf{- M g}$ & $\mathbf{- S}$ & -B \\
\hline $\mathrm{Ca}\left(\mathrm{NO}_{3}\right)_{2-} 4 \mathrm{H}_{2} \mathrm{O} 2 \mathrm{~mol} \mathrm{~L}^{-1}$ & 1 & & 1 & 1 & & 1 & 1 & 1 \\
$\mathrm{KNO}_{3} 2 \mathrm{~mol} \mathrm{~L}^{-1}$ & 2 & & 2 & & 2 & 2 & 2 & 2 \\
$\mathrm{MgSO}_{4} 7 \mathrm{H}_{2} \mathrm{O} 1 \mathrm{~mol} \mathrm{~L}^{-1}$ & 1 & 1 & 1 & 1 & 1 & & & 1 \\
$\left(\mathrm{NH}_{4}\right) \mathrm{H}_{2} \mathrm{PO}_{4} 1 \mathrm{~mol} \mathrm{~L}^{-1}$ & 0,25 & & & 0,25 & 0,25 & 0,25 & 0,25 & 0,25 \\
$\mathrm{H}_{3} \mathrm{BO}_{3}$ & 1 & 1 & 1 & 1 & 1 & 1 & 1 & \\
Fe-EDTA & 1 & 1 & 1 & 1 & 1 & 1 & 1 & 1 \\
$\mathrm{Micro}-\mathrm{B}-\mathrm{Fe}$ & 1 & 1 & 1 & 1 & 1 & 1 & 1 & 1 \\
$\mathrm{CaCl} 2 \mathrm{~mol} \mathrm{~L}^{-1}$ & & 1 & & & & & & \\
$\mathrm{NaNO}_{3} 2 \mathrm{~mol} \mathrm{~L}^{-1}$ & & & & 2 & 2 & & & \\
$\mathrm{KCL}_{1} 1 \mathrm{~mol} \mathrm{~L}^{-1}$ & & 3,75 & & & & & & \\
$\mathrm{Na}_{2} \mathrm{SO}_{4} 1 \mathrm{~mol} \mathrm{~L}^{-1}$ & & & & & & 1 & & \\
$\mathrm{KH}_{2} \mathrm{PO}_{4} 1 \mathrm{~mol} \mathrm{~L}^{-1}$ & & 0,25 & & & & & & \\
$\mathrm{MgCl}_{2} 1 \mathrm{~mol} \mathrm{~L}^{-1}$ & & & & & & & & \\
$\mathrm{NH}_{4} \mathrm{Cl}_{1} 1 \mathrm{~mol} \mathrm{~L}^{-1}$ & & & 0,25 & & & & &
\end{tabular}

*Para o preparo de 1L de solução de micronutrientes (-B -Fe), foram utilizados os seguintes reagentes analíticos em g: $\mathrm{KCl}$ : 3,7467; MnSO4 2 $\mathrm{H}_{2} \mathrm{O}: 0,8451 ; \mathrm{ZnSO}_{4} 7 \mathrm{H}_{2} \mathrm{O}: 0,5780 ; \mathrm{CuSO}_{4} 5 \mathrm{H}_{2} \mathrm{O}: 0,1267$ e $\left(\mathrm{NH}_{4}\right)_{6} \mathrm{Mo}_{7} \mathrm{O}_{24} 4 \mathrm{H}_{2} \mathrm{O}: 0,088$.

TABELA 2- Efeito da deficiência de $\mathrm{N}$ sobre os teores de $\mathrm{N}, \mathrm{P}, \mathrm{K}, \mathrm{Ca}, \mathrm{Mg}, \mathrm{S}\left(\mathrm{g} \mathrm{kg}^{-1}\right)$ e B (mg kg-1) na matéria seca da folha "D", em abacaxizeiro 'Imperial', em diferentes fases do ciclo da planta. Campos dos Goytacazes-RJ.

\begin{tabular}{lccccc}
\hline \multirow{2}{*}{ Tratamento } & \multirow{2}{*}{ Nutriente } & \multicolumn{4}{c}{ Meses após o plantio } \\
\cline { 3 - 6 } & & 5 & 7 & 9 & 12 \\
\cline { 3 - 6 } Completo & \multirow{2}{*}{$\mathrm{N}$} & $13,3 \mathrm{~A}$ & $14,8 \mathrm{~A}$ & $14,8 \mathrm{~A}$ & $13,4 \mathrm{~A}$ \\
-N & & $8,70 \mathrm{~A}-$ & $6,6 \mathrm{~B}-$ & $9,7 \mathrm{~A}-$ & $6,8 \mathrm{~B}-$ \\
\hline Completo & $\mathrm{P}$ & $1,30 \mathrm{~A}$ & $1,37 \mathrm{~A}$ & $1,23 \mathrm{AB}$ & $1,04 \mathrm{~B}$ \\
$-\mathrm{N}$ & & $1,50 \mathrm{Dns}$ & $2,00 \mathrm{C}+$ & $2,24 \mathrm{~B}+$ & $2,60 \mathrm{~A}+$ \\
\hline Completo & $\mathrm{K}$ & $21,6 \mathrm{AB}$ & $23,0 \mathrm{AB}$ & $20,0 \mathrm{~B}$ & $23,8 \mathrm{~A}$ \\
$-\mathrm{N}$ & & $26,6 \mathrm{C}+$ & $32,4 \mathrm{~B}+$ & $27,3 \mathrm{C}+$ & $43,3 \mathrm{~A}+$ \\
\hline Completo & $\mathrm{Ca}$ & $4,30 \mathrm{~B}$ & $4,40 \mathrm{~B}$ & $4,37 \mathrm{~B}$ & $7,59 \mathrm{~A}$ \\
$-\mathrm{N}$ & & $4,80 \mathrm{Bns}$ & $3,70 \mathrm{Bns}$ & $4,73 \mathrm{Bns}$ & $8,09 \mathrm{Ans}$ \\
\hline Completo & $\mathrm{Mg}$ & $2,10 \mathrm{~B}$ & $2,30 \mathrm{~B}$ & $2,26 \mathrm{~B}$ & $3,07 \mathrm{~A}$ \\
$-\mathrm{N}$ & & $2,50 \mathrm{Bns}$ & $2,70 \mathrm{Bns}$ & $2,50 \mathrm{Bns}$ & $4,08 \mathrm{~A}+$ \\
\hline Completo & \multirow{2}{*}{$\mathrm{S}$} & $1,80 \mathrm{~A}$ & $1,54 \mathrm{~A}$ & $1,45 \mathrm{~A}$ & $1,63 \mathrm{~A}$ \\
$-\mathrm{N}$ & & $1,40 \mathrm{~A}-$ & $1,18 \mathrm{AB}-$ & $1,24 \mathrm{ABns}$ & $1,01 \mathrm{~B}-$ \\
\hline Completo & \multirow{2}{*}{$\mathrm{B}$} & $21,2 \mathrm{~B}$ & $20,0 \mathrm{~B}$ & $18,4 \mathrm{~B}$ & $30,5 \mathrm{~A}$ \\
$-\mathrm{N}$ & & $25,4 \mathrm{~B}+$ & $22,0 \mathrm{Bns}$ & $24,3 \mathrm{~B}+$ & $39,4 \mathrm{~A}+$ \\
\hline
\end{tabular}

Médias seguidas pela mesma letra maiúscula na linha não diferem entre si, pelo teste de Tukey, a 5\%. Para cada nutriente, em cada coluna, média seguida por + , - ou ns é maior, menor ou não difere do tramento completo, respectivamente, pelo teste de Dunnett, a 5\%. 
TABELA 3- Efeito da deficiência de P sobre os teores de N, P, K, Ca, Mg, S ( $\left.\mathrm{g} \mathrm{kg}^{-1}\right)$ e B $\left(\mathrm{mg} \mathrm{kg}^{-1}\right)$ na matéria seca da folha "D", em abacaxizeiro 'Imperial', em diferentes fases do ciclo da planta.Campos dos Goytacazes-RJ.

\begin{tabular}{lccccc}
\hline \multirow{2}{*}{ Tratamento } & \multirow{2}{*}{ Nutriente } & \multicolumn{4}{c}{ Meses após o plantio } \\
\cline { 3 - 6 } & & 5 & 7 & 9 & 12 \\
\hline Completo & $\mathrm{N}$ & $13,3 \mathrm{~A}$ & $14,8 \mathrm{~A}$ & $14,8 \mathrm{~A}$ & $13,4 \mathrm{~A}$ \\
$-\mathrm{P}$ & & $13,3 \mathrm{Ans}$ & $12,5 \mathrm{~A}-$ & $12,8 \mathrm{~A}-$ & $11,8 \mathrm{~A}-$ \\
\hline Completo & $\mathrm{P}$ & $1,30 \mathrm{~A}$ & $1,37 \mathrm{~A}$ & $1,23 \mathrm{AB}$ & $1,04 \mathrm{~B}$ \\
$-\mathrm{P}$ & & $0,97 \mathrm{~A}-$ & $0,70 \mathrm{~B}-$ & $0,67 \mathrm{~B}-$ & $0,32 \mathrm{C}-$ \\
\hline Completo & $\mathrm{K}$ & $21,6 \mathrm{AB}$ & $23,0 \mathrm{AB}$ & $20,0 \mathrm{~B}$ & $23,8 \mathrm{~A}$ \\
$-\mathrm{P}$ & & $21,6 \mathrm{BCns}$ & $24,0 \mathrm{Bns}$ & $20,3 \mathrm{Cnn}$ & $29,2 \mathrm{~A}+$ \\
\hline Completo & $\mathrm{Ca}$ & $4,30 \mathrm{~B}$ & $4,40 \mathrm{~B}$ & $4,37 \mathrm{~B}$ & $7,59 \mathrm{~A}$ \\
$-\mathrm{P}$ & & $4,60 \mathrm{Bns}$ & $3,30 \mathrm{Bns}$ & $3,40 \mathrm{Bns}$ & $5,55 \mathrm{~A}-$ \\
\hline Completo & $\mathrm{Mg}$ & $2,10 \mathrm{~B}$ & $2,30 \mathrm{~B}$ & $2,26 \mathrm{~B}$ & $3,07 \mathrm{~A}$ \\
$-\mathrm{P}$ & & $2,10 \mathrm{Bns}$ & $2,00 \mathrm{Bns}$ & $2,12 \mathrm{Bns}$ & $2,60 \mathrm{~A}-$ \\
\hline Completo & $\mathrm{S}$ & $1,80 \mathrm{~A}$ & $1,54 \mathrm{~A}$ & $1,45 \mathrm{~A}$ & $1,63 \mathrm{~A}$ \\
$-\mathrm{P}$ & & $1,60 \mathrm{Ans}$ & $1,12 \mathrm{~B}-$ & $0,89 \mathrm{~B}-$ & $0,96 \mathrm{~B}-$ \\
\hline Completo & $\mathrm{B}$ & $21,2 \mathrm{~B}$ & $20,0 \mathrm{~B}$ & $18,4 \mathrm{~B}$ & $30,5 \mathrm{~A}$ \\
$-\mathrm{P}$ & & $21,5 \mathrm{Ans}$ & $17,6 \mathrm{Bns}$ & $15,0 \mathrm{~B}-$ & $22,5 \mathrm{~A}-$ \\
\hline
\end{tabular}

Médias seguidas pela mesma letra maiúscula na linha não diferem entre si, pelo teste de Tukey, a 5\%. Para cada nutriente, em cada coluna, média seguida por +, - ou ns é maior, menor ou não difere do tratamento completo, respectivamente, pelo teste de Dunnett, a $5 \%$.

TABELA 4- Efeito da deficiência de $\mathrm{K}$ sobre os teores de N, $\mathrm{P}, \mathrm{K}, \mathrm{Ca}, \mathrm{Mg}, \mathrm{S}\left(\mathrm{g} \mathrm{kg}^{-1}\right)$ e B (mg kg-1) na matéria seca da folha "D", em abacaxizeiro 'Imperial', em diferentes fases do ciclo da planta. Campos dos Goytacazes-RJ.

\begin{tabular}{lccccc}
\hline \multirow{2}{*}{ Tratamento } & \multirow{2}{*}{ Nutriente } & \multicolumn{4}{c}{ Meses após o plantio } \\
\cline { 3 - 6 } & & 5 & 7 & 9 & 12 \\
\hline Completo & $\mathrm{N}$ & $13,3 \mathrm{~A}$ & $14,8 \mathrm{~A}$ & $14,8 \mathrm{~A}$ & $13,4 \mathrm{~A}$ \\
$-\mathrm{K}$ & & $14,6 \mathrm{Bns}$ & $16,5 \mathrm{~A}+$ & $17,8 \mathrm{~A}+$ & $16,6 \mathrm{~A}+$ \\
\hline Completo & $\mathrm{P}$ & $1,30 \mathrm{~A}$ & $1,37 \mathrm{~A}$ & $1,23 \mathrm{AB}$ & $1,04 \mathrm{~B}$ \\
$-\mathrm{K}$ & & $1,40 \mathrm{Ans}$ & $1,50 \mathrm{Ans}$ & $1,53 \mathrm{~A}+$ & $1,39 \mathrm{~A}+$ \\
\hline Completo & $\mathrm{K}$ & $21,6 \mathrm{AB}$ & $23,0 \mathrm{AB}$ & $20,0 \mathrm{~B}$ & $23,8 \mathrm{~A}$ \\
$-\mathrm{K}$ & & $13,8 \mathrm{~A}-$ & $11,6 \mathrm{~B}-$ & $11,6 \mathrm{~B}-$ & $3,2 \mathrm{C}-$ \\
\hline Completo & $\mathrm{Ca}$ & $4,30 \mathrm{~B}$ & $4,40 \mathrm{~B}$ & $4,37 \mathrm{~B}$ & $7,59 \mathrm{~A}$ \\
$-\mathrm{K}$ & & $5,30 \mathrm{Bns}$ & $6,20 \mathrm{~B}+$ & $5,76 \mathrm{~B}+$ & $14,9 \mathrm{~A}+$ \\
\hline Completo & $\mathrm{Mg}$ & $2,10 \mathrm{~B}$ & $2,30 \mathrm{~B}$ & $2,26 \mathrm{~B}$ & $3,07 \mathrm{~A}$ \\
$-\mathrm{K}$ & & $2,60 \mathrm{~B}+$ & $3,00 \mathrm{~B}+$ & $2,76 \mathrm{~B}+$ & $5,16 \mathrm{~A}+$ \\
\hline Completo & $\mathrm{S}$ & $1,80 \mathrm{~A}$ & $1,54 \mathrm{~A}$ & $1,45 \mathrm{~A}$ & $1,63 \mathrm{~A}$ \\
$-\mathrm{K}$ & & $1,90 \mathrm{Bns}$ & $1,81 \mathrm{Bns}$ & $1,61 \mathrm{Bns}$ & $2,26 \mathrm{~A}+$ \\
\hline Completo & $\mathrm{B}$ & $21,2 \mathrm{~B}$ & $20,0 \mathrm{~B}$ & $18,4 \mathrm{~B}$ & $30,5 \mathrm{~A}$ \\
$-\mathrm{K}$ & & $21,9 \mathrm{Bns}$ & $21,6 \mathrm{Bns}$ & $19,2 \mathrm{Bns}$ & $37,1 \mathrm{~A}+$ \\
\hline
\end{tabular}

Médias seguidas pela mesma letra maiúscula na linha não diferem entre si, pelo teste de Tukey, a 5\%.

Para cada nutriente, em cada coluna, média seguida por + , - ou ns é maior, menor ou não difere do

tratamento completo, respectivamente, pelo teste de Dunnett, a 5\%. 
TABELA 5- Efeito da deficiência de Ca sobre os teores de $\mathrm{N}, \mathrm{P}, \mathrm{K}, \mathrm{Ca}, \mathrm{Mg}, \mathrm{S}\left(\mathrm{g} \mathrm{kg}^{-1}\right)$ e B $\left(\mathrm{mg} \mathrm{kg}^{-1}\right) \mathrm{na}$ matéria seca da folha " $D$ ", em abacaxizeiro 'Imperial', em diferentes fases do ciclo da planta. Campos dos Goytacazes-RJ.

\begin{tabular}{lccccc}
\hline \multirow{2}{*}{ Tratamento } & \multirow{2}{*}{ Nutriente } & \multicolumn{4}{c}{ Meses após o plantio } \\
\cline { 3 - 6 } Completo & $\mathrm{N}$ & $13,3 \mathrm{~A}$ & $14,8 \mathrm{~A}$ & $14,8 \mathrm{~A}$ & $13,4 \mathrm{~A}$ \\
-Ca & & $13,2 \mathrm{Bns}$ & $15,4 \mathrm{Ans}$ & $15,4 \mathrm{Ans}$ & $12,8 \mathrm{Bns}$ \\
\hline Completo & $\mathrm{P}$ & $1,30 \mathrm{~A}$ & $1,37 \mathrm{~A}$ & $1,23 \mathrm{AB}$ & $1,04 \mathrm{~B}$ \\
$-\mathrm{Ca}$ & & $1,40 \mathrm{ABns}$ & $1,48 \mathrm{Ans}$ & $1,24 \mathrm{BCns}$ & $1,05 \mathrm{Cns}$ \\
\hline Completo & $\mathrm{K}$ & $21,6 \mathrm{AB}$ & $23,0 \mathrm{AB}$ & $20,0 \mathrm{~B}$ & $23,8 \mathrm{~A}$ \\
$-\mathrm{Ca}$ & & $22,5 \mathrm{Bns}$ & $26,9 \mathrm{~A}+$ & $21,2 \mathrm{Bns}$ & $26,6 \mathrm{~A}+$ \\
\hline Completo & $\mathrm{Ca}$ & $4,30 \mathrm{~B}$ & $4,40 \mathrm{~B}$ & $4,37 \mathrm{~B}$ & $7,59 \mathrm{~A}$ \\
$-\mathrm{Ca}$ & & $2,30 \mathrm{~A}-$ & $1,30 \mathrm{AB}-$ & $0,91 \mathrm{~B}-$ & $1,72 \mathrm{AB}-$ \\
\hline Completo & $\mathrm{Mg}$ & $2,10 \mathrm{~B}$ & $2,30 \mathrm{~B}$ & $2,26 \mathrm{~B}$ & $3,07 \mathrm{~A}$ \\
$-\mathrm{Ca}$ & & $2,20 \mathrm{Cns}$ & $3,00 \mathrm{~B}+$ & $2,84 \mathrm{~B}+$ & $3,50 \mathrm{~A}+$ \\
\hline Completo & $\mathrm{S}$ & $1,80 \mathrm{~A}$ & $1,54 \mathrm{~A}$ & $1,45 \mathrm{~A}$ & $1,63 \mathrm{~A}$ \\
$-\mathrm{Ca}$ & & $1,70 \mathrm{Ans}$ & $1,59 \mathrm{ABns}$ & $1,18 \mathrm{Bns}$ & $1,30 \mathrm{~B}-$ \\
\hline Completo & $\mathrm{B}$ & $21,2 \mathrm{~B}$ & $20,0 \mathrm{~B}$ & $18,4 \mathrm{~B}$ & $30,5 \mathrm{~A}$ \\
$-\mathrm{Ca}$ & & $21,7 \mathrm{Bns}$ & $20,6 \mathrm{BCns}$ & $17,6 \mathrm{Cns}$ & $30,9 \mathrm{Ans}$ \\
\hline
\end{tabular}

Médias seguidas pela mesma letra maiúscula na linha não diferem entre si, pelo teste de Tukey, a 5\%. Para cada nutriente, em cada coluna, média seguida por,+ - ou ns é maior, menor ou não difere do tratamento completo, respectivamente, pelo teste de Dunnett, a $5 \%$.

TABELA 6- Efeito da deficiência de $\mathrm{Mg}$ sobre os teores de $\mathrm{N}, \mathrm{P}, \mathrm{K}, \mathrm{Ca}, \mathrm{Mg}, \mathrm{S}\left(\mathrm{g} \mathrm{kg}^{-1}\right)$ e B $\left(\mathrm{mg} \mathrm{kg}^{-1}\right)$ na matéria seca da folha " $\mathrm{D}$ ", em abacaxizeiro 'Imperial', em diferentes fases do ciclo da planta. Campos dos Goytacazes-RJ.

\begin{tabular}{lccccc}
\hline \multirow{2}{*}{ Tratamento } & \multirow{2}{*}{ Nutriente } & \multicolumn{4}{c}{ Meses após o plantio } \\
\cline { 3 - 6 } & & 5 & 7 & 9 & 12 \\
\hline Completo & $\mathrm{N}$ & $13,3 \mathrm{~A}$ & $14,8 \mathrm{~A}$ & $14,8 \mathrm{~A}$ & $13,4 \mathrm{~A}$ \\
$-\mathrm{Mg}$ & & $14,5 \mathrm{ABns}$ & $15,2 \mathrm{ABns}$ & $15,9 \mathrm{Ans}$ & $14,3 \mathrm{Bns}$ \\
\hline Completo & $\mathrm{P}$ & $1,30 \mathrm{~A}$ & $1,37 \mathrm{~A}$ & $1,23 \mathrm{AB}$ & $1,04 \mathrm{~B}$ \\
$-\mathrm{Mg}$ & & $1,50 \mathrm{Ans}$ & $1,40 \mathrm{Ans}$ & $1,45 \mathrm{~A}+$ & $1,17 \mathrm{Bns}$ \\
\hline Completo & $\mathrm{K}$ & $21,6 \mathrm{AB}$ & $23,0 \mathrm{AB}$ & $20,0 \mathrm{~B}$ & $23,8 \mathrm{~A}$ \\
$-\mathrm{Mg}$ & & $22,8 \mathrm{Bns}$ & $26,3 \mathrm{~A}+$ & $22,7 \mathrm{Bns}$ & $25,9 \mathrm{Ans}$ \\
\hline Completo & $\mathrm{Ca}$ & $4,30 \mathrm{~B}$ & $4,40 \mathrm{~B}$ & $4,37 \mathrm{~B}$ & $7,59 \mathrm{~A}$ \\
$-\mathrm{Mg}$ & & $5,50 \mathrm{~B}+$ & $5,40 \mathrm{Bns}$ & $6,32 \mathrm{~B}+$ & $10,70 \mathrm{~A}+$ \\
\hline Completo & $\mathrm{Mg}$ & $2,10 \mathrm{~B}$ & $2,30 \mathrm{~B}$ & $2,26 \mathrm{~B}$ & $3,07 \mathrm{~A}$ \\
$-\mathrm{Mg}$ & & $1,30 \mathrm{~A}-$ & $0,90 \mathrm{AB}-$ & $0,73 \mathrm{~B}-$ & $0,54 \mathrm{~B}-$ \\
\hline Completo & $\mathrm{S}$ & $1,80 \mathrm{~A}$ & $1,54 \mathrm{~A}$ & $1,45 \mathrm{~A}$ & $1,63 \mathrm{~A}$ \\
$-\mathrm{Mg}$ & & $1,70 \mathrm{Ans}$ & $1,75 \mathrm{Ans}$ & $1,65 \mathrm{Ans}$ & $1,82 \mathrm{Ans}$ \\
\hline Completo & $\mathrm{B}$ & $21,2 \mathrm{~B}$ & $20,0 \mathrm{~B}$ & $18,4 \mathrm{~B}$ & $30,5 \mathrm{~A}$ \\
$-\mathrm{Mg}$ & & $22,5 \mathrm{Bns}$ & $21,5 \mathrm{Bns}$ & $19,4 \mathrm{Bns}$ & $33,0 \mathrm{Ans}$ \\
\hline
\end{tabular}

Médias seguidas pela mesma letra maiúscula na linha não diferem entre si, pelo teste de Tukey, a 5\%. Para cada nutriente, em cada coluna, média seguida por + , - ou ns é maior, menor ou não difere do tratamento completo, respectivamente, pelo teste de Dunnett, a $5 \%$. 
TABELA 7-.Efeito da deficiência de $\mathrm{S}$ sobre os teores de $\mathrm{N}, \mathrm{P}, \mathrm{K}, \mathrm{Ca}, \mathrm{Mg}, \mathrm{S}\left(\mathrm{g} \mathrm{kg}^{-1}\right)$ e B $\left(\mathrm{mg} \mathrm{kg}^{-1}\right)$ na matéria seca da folha "D", em abacaxizeiro 'Imperial', em diferentes fases do ciclo da planta.Campos dos Goytacazes-RJ.

\begin{tabular}{lccccc}
\hline \multirow{2}{*}{ Tratamento } & \multirow{2}{*}{ Nutriente } & \multicolumn{4}{c}{ Meses após o plantio } \\
\cline { 3 - 6 } & & 5 & 7 & 9 & 12 \\
\hline Completo & $\mathrm{N}$ & $13,3 \mathrm{~A}$ & $14,8 \mathrm{~A}$ & $14,8 \mathrm{~A}$ & $13,4 \mathrm{~A}$ \\
$-\mathrm{S}$ & & $13,9 \mathrm{ABns}$ & $14,6 \mathrm{Ans}$ & $15,3 \mathrm{Ans}$ & $13,0 \mathrm{Bns}$ \\
\hline Completo & $\mathrm{P}$ & $1,30 \mathrm{~A}$ & $1,37 \mathrm{~A}$ & $1,23 \mathrm{AB}$ & $1,04 \mathrm{~B}$ \\
$-\mathrm{S}$ & & $1,40 \mathrm{Ans}$ & $1,40 \mathrm{Ans}$ & $1,48 \mathrm{~A}+$ & $1,16 \mathrm{Bns}$ \\
\hline Completo & $\mathrm{K}$ & $21,6 \mathrm{AB}$ & $23,0 \mathrm{AB}$ & $20,0 \mathrm{~B}$ & $23,8 \mathrm{~A}$ \\
$-\mathrm{S}$ & & $22,5 \mathrm{Bns}$ & $26,0 \mathrm{~A}+$ & $20,7 \mathrm{Bns}$ & $26,9 \mathrm{~A}+$ \\
\hline Completo & $\mathrm{Ca}$ & $4,30 \mathrm{~B}$ & $4,40 \mathrm{~B}$ & $4,37 \mathrm{~B}$ & $7,59 \mathrm{~A}$ \\
$-\mathrm{S}$ & & $5,40 \mathrm{Bns}$ & $4,60 \mathrm{Bns}$ & $4,84 \mathrm{Bns}$ & $9,15 \mathrm{~A}+$ \\
\hline Completo & $\mathrm{Mg}$ & $2,10 \mathrm{~B}$ & $2,30 \mathrm{~B}$ & $2,26 \mathrm{~B}$ & $3,07 \mathrm{~A}$ \\
$-\mathrm{S}$ & & $1,90 \mathrm{Bns}$ & $2,30 \mathrm{Bns}$ & $2,32 \mathrm{Bns}$ & $3,30 \mathrm{Ans}$ \\
\hline Completo & $\mathrm{S}$ & $1,80 \mathrm{~A}$ & $1,54 \mathrm{~A}$ & $1,45 \mathrm{~A}$ & $1,63 \mathrm{~A}$ \\
$-\mathrm{S}$ & & $1,20 \mathrm{~A}-$ & $0,56 \mathrm{~B}-$ & $0,45 \mathrm{~B}-$ & $0,54 \mathrm{~B}-$ \\
\hline Completo & $\mathrm{B}$ & $21,2 \mathrm{~B}$ & $20,0 \mathrm{~B}$ & $18,4 \mathrm{~B}$ & $30,5 \mathrm{~A}$ \\
$-\mathrm{S}$ & & $23,2 \mathrm{Bns}$ & $22,2 \mathrm{BCns}$ & $19,2 \mathrm{Cns}$ & $35,2 \mathrm{~A}+$ \\
\hline
\end{tabular}

Médias seguidas pela mesma letra maiúscula na linha não diferem entre si, pelo teste de Tukey, a $5 \%$.

Para cada nutriente, em cada coluna, média seguida por + , - ou ns é maior, menor ou não difere do tratamento completo, respectivamente, pelo teste de Dunnett, a $5 \%$.

TABELA 8- Efeito da deficiência de B sobre os teores de N, $\mathrm{P}, \mathrm{K}, \mathrm{Ca}, \mathrm{Mg}, \mathrm{S}\left(\mathrm{g} \mathrm{kg}^{-1}\right)$ e B (mg kg-1) na matéria seca da folha "D", em abacaxizeiro 'Imperial', em diferentes fases do ciclo da planta. Campos dos Goytacazes-RJ.

\begin{tabular}{lccccc}
\hline \multirow{2}{*}{ Tratamento } & \multirow{2}{*}{ Nutriente } & \multicolumn{4}{c}{ Meses após o plantio } \\
\cline { 3 - 6 } & & 5 & 7 & 9 & 12 \\
\hline Completo & $\mathrm{N}$ & $13,3 \mathrm{~A}$ & $14,8 \mathrm{~A}$ & $14,8 \mathrm{~A}$ & $13,4 \mathrm{~A}$ \\
-B & & $14,0 \mathrm{ABns}$ & $15,2 \mathrm{Ans}$ & $15,0 \mathrm{ABns}$ & $13,6 \mathrm{Bns}$ \\
\hline Completo & $\mathrm{P}$ & $1,30 \mathrm{~A}$ & $1,37 \mathrm{~A}$ & $1,23 \mathrm{AB}$ & $1,04 \mathrm{~B}$ \\
-B & & $1,40 \mathrm{Ans}$ & $1,40 \mathrm{Ans}$ & $1,23 \mathrm{Ans}$ & $0,95 \mathrm{Bns}$ \\
\hline Completo & $\mathrm{K}$ & $21,6 \mathrm{AB}$ & $23,0 \mathrm{AB}$ & $20,0 \mathrm{~B}$ & $23,8 \mathrm{~A}$ \\
-B & & $22,3 \mathrm{ABns}$ & $25,2 \mathrm{Ans}$ & $20,3 \mathrm{Bns}$ & $24,1 \mathrm{Ans}$ \\
\hline Completo & $\mathrm{Ca}$ & $4,30 \mathrm{~B}$ & $4,40 \mathrm{~B}$ & $4,37 \mathrm{~B}$ & $7,59 \mathrm{~A}$ \\
$-\mathrm{B}$ & & $4,50 \mathrm{Bns}$ & $4,20 \mathrm{Bns}$ & $4,87 \mathrm{Bns}$ & $9,10 \mathrm{~A}+$ \\
\hline Completo & $\mathrm{Mg}$ & $2,10 \mathrm{~B}$ & $2,30 \mathrm{~B}$ & $2,26 \mathrm{~B}$ & $3,07 \mathrm{~A}$ \\
$-\mathrm{B}$ & & $2,00 \mathrm{Bns}$ & $2,20 \mathrm{Bns}$ & $2,34 \mathrm{Bns}$ & $3,11 \mathrm{Ans}$ \\
\hline Completo & $\mathrm{S}$ & $1,80 \mathrm{~A}$ & $1,54 \mathrm{~A}$ & $1,45 \mathrm{~A}$ & $1,63 \mathrm{~A}$ \\
$-\mathrm{B}$ & & $1,90 \mathrm{Ans}$ & $1,90 \mathrm{~A}+$ & $1,60 \mathrm{Ans}$ & $1,71 \mathrm{Ans}$ \\
\hline Completo & $\mathrm{B}$ & $21,2 \mathrm{~B}$ & $20,0 \mathrm{~B}$ & $18,4 \mathrm{~B}$ & $30,5 \mathrm{~A}$ \\
$-\mathrm{B}$ & & $8,5 \mathrm{~A}-$ & $5,6 \mathrm{~A}-$ & $5,8 \mathrm{~A}-$ & $5,5 \mathrm{~A}-$ \\
\hline
\end{tabular}

Médias seguidas pela mesma letra maiúscula na linha não diferem entre si,pelo teste de Tukey, a 5\%.

Para cada nutriente, em cada coluna, média seguida por + , - ou ns é maior, menor ou não difere do

Tratamento completo, respectivamente, pelo teste de Dunnett, a $5 \%$. 


\section{CONCLUSÕES}

1 - A deficiência de cada nutriente reduz seu próprio teor foliar em todas as épocas.

2- A deficiência de $\mathrm{N}$ reduz a absorção de enxofre, mas aumenta os teores foliares de P, K, $\mathrm{Mg}$ e B.

3- A deficiência de $\mathrm{P}$ reduz os teores foliares de $\mathrm{N}, \mathrm{Ca}, \mathrm{Mg}, \mathrm{S}$ e $\mathrm{B}$, mas aumenta os de $\mathrm{K}$.

4- Os teores foliares de N, P, Ca, Mg, S e B aumentam nas plantas deficientes de $\mathrm{K}$.

5- A deficiência de $\mathrm{Ca}$ aumenta os teores foliares de $\mathrm{K}$ e $\mathrm{Mg}$, mas reduz os de $\mathrm{S}$; a de $\mathrm{Mg}$ aumenta os de $\mathrm{Ca}$; a de $\mathrm{S}$ aumenta os de $\mathrm{K}$ e $\mathrm{B}$, e a de $\mathrm{B}$ aumenta os de $\mathrm{Ca}$.

6- Os teores foliares das plantas normais e deficientes, aos 7 meses, são: $\mathrm{N}=14,8$ e 6,$6 ; \mathrm{P}=1,37$ e 0,$70 ; \mathrm{K}=23,0$ e 11,$6 ; \mathrm{Ca}=4,40$ e 1,$30 ; \mathrm{MG}=2,30$ e 0,$90 ; \mathrm{S}=1,54$ e $0,56 \mathrm{~g} \mathrm{~kg}^{-1}$ e $\mathrm{B}=20,0$ e $5,6 \mathrm{mg} \mathrm{kg}^{-1}$.

7- Os teores foliares das plantas normais e deficientes no início da formação dos frutos são, respectivamente: $\mathrm{N}=14,8$ e 9,$7 ; \mathrm{P}=1,23$ e 0,67 ; $\mathrm{K}=20,0$ e 11,$6 ; \mathrm{Ca}=4,37$ e 0,$91 ; \mathrm{Mg}=2,26$ e 0,73 ; $\mathrm{S}=1,45$ e $0,45 \mathrm{~g} \mathrm{~kg}^{-1}$ e $\mathrm{B}=18,4$ e $5,8 \mathrm{mg} \mathrm{kg}^{-1}$.

\section{REFERÊNCIAS}

AVILÁN, L.R. Efecto de la omisión de los macronutrientes en el desarollo y composición química de la guanábana (Annona muricata L.) cultivada en soluciones nutritivas. Agronomia Tropical, Macaray, v. 25, p. $73-79,1975$.

BATAGLIA, O.C.; SANTOS, W.R. Estado nutricional de plantas perenes: avaliação e monitoramento. Informações Agronômicas, Piracicaba, n.96, p.1-8, 2001.

BOARETTO, A.; CHITOLINA, J.C.; RAIJ, B.Van; SILVA, F.C.; TEDESCO, M.J.; CARMO, C.A.F.S. Amostragem, acondicionamento e preparação das amostras de plantas para análise química. In: SILVA, F.C (Org.). Manual de análises químicas de solos, plantas e fertilizantes. Brasília: Embrapa Comunicação para a Transferência de Tecnologia, 1999. p.49-74
EMBRAPA. Manual de análises químicas de solos, plantas e fertilizantes. Brasília. 1999, 370p.

EPSTEIN, E.; BLOOM, A.J. Nutrição mineral de plantas: princípios e perspectivas. 2. ed. Londrina: Editora Planta, 2006. 403p.

FONTES, P.C.R. Diagnóstico do estado nutricional pelo método visual. In: FONTES, P.C. R. (Ed.). Diagnóstico do estado nutricional das plantas. Viçosa: UFV, 2001. p.63-86.

FRAZÃO, D.A.C.; VIÉGAS I.J.M.; BATISTA M.M.F.; CRUZ, E.S.; SILVA, J.F. Teores de N, P, K, $\mathrm{Ca}, \mathrm{Mg}$ e $\mathrm{S}$ em Gravioleiras cultivadas em solução nutritiva com omissão de macronutrientes. In: $\mathrm{CON}$ GRESSO BRASILEIRO DE FRUTICULTURA, 17. 2002. Belém. Anais... CD-ROM

HIROCE, R.; BATAGLIA, O.C.; FURLANI, P.R.; FURLANI, A.M.C.; GIACOMELLI, E.J.; GALLO, J.R. Composição química inorgânica do abacaxizeiro (Ananas comosus (L.) Merr.), cv.Smooth Cayenne da região de Bebedouro-SP. Ciência e Cultura, São Paulo, v. 29, p.323-326, 1977.

JACKSON, M.L. Soil chemical analysis. New Jersey: Prentice Hall, 1965. 498 p.

JONES, Jr. J.B.; WOLF, B.; MILLS, H.A. Plant analysis handbook: a pratical sampling, preparation, analysis, and interpretation guide. Athens: MicroMacro Publishing, 1991. 213 p.

KLUGE, R.A.; FILHO, J.A.S.; JACOMINO, A.P.; PEIXOTO, C.P. Distúrbios fisiológicos em frutos. Piracicaba: FEALQ, 2001. 58p.

KOPRIVOVA, A.; SUTER, M.; ROEL, O.C.; BRUNOLD, C.; KOPRIVA, S. Regulation of sulfate assimilation by nitrogen in Arabidopsis. Plant Physiology, Washington, p.737-746, 2000.

MALAVOLTA, E.; VITTI, G.C.; OLIVEIRA, S.A Avaliação do estado nutricional das plantas: princípios e aplicações, 2.ed. Piracicaba: POTAFÓS, 1997. 319p.

MALÉZIEUX, E.; BARTHOLOMEW, D.P. Plant Nutrition. In:BARTHOLOMEW, D.P.; PAUL, R.E, ROHRBACH, K.G. (Ed.). The pineapple: botany, production and uses. New York: CABI Publishing, 2003. p. 143-165. 
MARSCHNER, H. Mineral nutrition of higher plants. 2.ed. London: Academic Press, 1995. 675p.

NEUENSCHWANDER, U.; SUTER, M.; BRUNOLD, C. Regulation of sulfate assimilation by light and O-acetyl-1-serine in Lemna minor L. Plant Physiology,Washington, v.97, p. 253-258, 1991.

PAULA, M.B.; HOLANDA, F.S.R.; MESQUITA, H.A.; CARVALHO, V.D. Uso da vinhaça no abacaxizeiro em solo de baixo potencial de produção. Pesquisa Agropecuária Brasileira, Brasília, v.34, n. 7, p.1217-1222, 1999.

PAULA, M.B.; MESQUITA, H.A.; NOGUEIRA, F.D. Nutrição e adubação do abacaxizeiro. Informe Agropecuário, Belo Horizonte, v. 19, n.185, p. 3339, 1998.

QUAGGIO, J.A.; RAIJ, B.Van.; PIZA JR., C.T. Frutíferas. In: RAIJ, B. Van et al. (Eds.), Recomendações de adubação e calagem para o Estado de São Paulo. 2.ed. Campinas: IAC, 1996. p.121-125. (Boletim Técnico, 100)

RAMOS, M. J. M.; MONNERAT, H. P.; CARVALHO, A. J. C. de, Pinto, J. L. A., SILVA, J. A. da. Sintomas visuais de deficiência de macronutrientes e de boro em abacaxizeiro 'imperial'. Revista Brasileira de Fruticultura, Jaboticabal, v. 31, n. 1, p.252-256, 2009.

REUTER, D.J.; ROBINSON, J.B. Plant analysis $3 / 4$ na interpretation manual. Melbourne: Inkata Press, 1988. 218 p.
SIEBENEICHLER, S.C. O boro na cultura do abacaxizeiro 'Pérola' no norte do Estado do Rio de Janeiro. 2002. 75f. Tese (Doutorado Solos e Nutrição de Plantas) - Universidade Estadual do Norte Fluminense Darcy Ribeiro, Campos dos Goyatacazes, 2002.

SILVA, H.; SILVA, A.Q.; ROQUE, M.L.; MALAVOLTA, E. Nutrição Mineral da Graviola (Annona muricata L). II. Teores de macronutrientes e boro. In: CONGRESSO BRASILEIRO DE FRUTICULTURA. 8., 1986, Brasília. Anais... p.303-307.

SOUZA, L.F.S.; REINHARDT, D.H. A adubação do abacaxizeiro após a indução floral. 2004. Disponível: $<$ http://www.cnpmf.Br/publicações/comunicados/ comunicado-103.pdf $>$. Acesso em: 15 set. 2007.

VELOSO, C.A.C.; OEIRAS, A.H.L.;CARVALHO, E.J.M.; SOUZA, F.R.S. Resposta do abacaxizeiro à adição de nitrogênio, potássio e calcário em latossolo amarelo do nordeste paraense. Revista Brasileira Fruticultura, Jaboticabal, v. 23, p. 396-402, 2001.

VIEGAS, I.J.M.; THOMAZ, M.A.A.; SILVA, J.F.; CONCEIÇÃO, E.O.; NAIFF, A.P.M. Efeito da omissão de macronutrientes e boro no crescimento, nos sintomas de deficiências nutricionais e na composição mineral de plantas de camucamuzeiro. Revista Brasileira Fruticultura, Jaboticabal, v. 26, n. 2, p. 315-319, 2004. 